\title{
The Hox transcription factor Ubx ensures somatic myogenesis by suppressing the mesodermal master regulator Twist
}

\author{
Katrin Domsch $\ddagger$ \\ Julia Schröder \\ Matthias Janeschik \\ Christoph Schaub \\ Ingrid Lohmann
}

¥Author for correspondence e-mail: katrin.domsch@cos.uni-heidelberg.de (K.D.)

Keywords: Hox genes, Ultrabithorax (Ubx), mesoderm, twist, repression, Tinman, Tin, Mef2

\section{Abbreviations:}

Mailing address of corresponding author:
Katrin Domsch
Heidelberg University
Im Neuenheimer Feld 230
D-69120 Heidelberg

Germany

$\mathrm{PH}: \quad+496221-545519$

FX: $+496221-546424$

EM: katrin.domsch@cos.uni-heidelberg.de 


\begin{abstract}
Early determination factors and lineage-specific master regulators are essential for the specification of cell and tissue types. However, once a cell has committed to a specific fate, it is equally critical to restrict the activity of such factors to enable proper differentiation. In many studies the functional network for master regulators are under constant investigations. Yet, how these factors are silenced remains unclear. Using the Drosophila mesoderm as a model and a comparative genomic approach, we identified the Hox transcription factor (TF) Ultrabithorax (Ubx) to be critical for the repression of the mesodermal master regulator Twist (Twi). Mesoderm-specific Ubx loss-of-function experiments using CRISPR/Cas9 as well as overexpression experiments demonstrated that Ubx majorly impacts twi transcription. A detailed mechanistic analysis revealed that Ubx requires the function of the NK-homeodomain protein Tinman (Tin) but not the muscle differentiation factor Myocyte enhancer factor 2 (Mef2) to bind to the twi promoter. Furthermore, we found these TF interactions to be critical for silencing of the twi promoter region by recruiting the Polycomb DNA binding protein Pleiohomeotic (Pho). In sum, our study demonstrates that the Hox TF Ubx is a critical player in mediating the silencing of the mesodermal master regulator Twi, which is crucial for coordinated muscle differentiation.
\end{abstract}




\section{Introduction}

The early development of an organism is tightly regulated by networks of transcription factors (TFs) that ensure axis orientation, germ layer determination and cell type specification as well as differentiation. Some of these TFs, so-called master regulators, play fundamental roles in cell and tissue specification, as they act at the top of hierarchical cascades driving cell fate decisions (Levine and Davidson, 2005). Thus, many studies focus on understanding the role of these master regulators during cell type specification (Jin et al., 2013; Liu et al., 2009; Sandmann et al., 2007). However, early master regulators lose their functional importance once cells have decided on their developmental path, and they need to be inactivated to enable proper cell and tissue differentiation (Davis and Rebay, 2017). Despite this knowledge, the mechanisms driving the inactivation of early master regulators, which is critical for tissue development, are not well understood.

The evolutionary conserved class of Hox TFs represent ideal candidates to coordinate late specification and differentiation events through the inactivation of master regulators for various reasons. First, they function after cell fates have been specified and they are active throughout the life time of an organism (Pearson et al., 2005). And second, they are essential for segment specification along the anterior-posterior axis and are expressed in all tissues within one segment. Thereby, they endow cells not only with a segment-specific code but also perform tissue- and cell-specific functions (Akam, 1987; Graham et al., 1989; Lewis, 1978; Scott and Carroll, 1987). In this study, we focussed on the Hox TF Ultrabithorax (Ubx), a Hox TF which is essential for the specification of the last thoracic and the abdominal segments (Lewis, 1978). Previous genome-wide investigation of tissue-specific Hox function identified Ubx as a repressor of alternative cell fates in the mesoderm, which required the cooperation with the Polycomb complex (Domsch et al., 2019). Thus, we assumed that Ubx could coordinate muscle differentiation by repressing not only alternative fate genes but also mesodermal master regulators.

Development of the Drosophila mesoderm depends on the nuclear translocation of dorsal (dl), a TF activating the mesodermal master regulator Twist (Twi) (Jiang et al., 1991; Thisse et al., 1991). Twi in turn induces the expression of additional cell-type specific factors, including the NK-homeodomain TF Tinman (Tin) and the MADS-Box TF Myocyte enhancer factor 2 (Mef2) (Cripps et al., 1998; Sandmann et al., 2007; Yin et al., 1997). Tin is the critical for visceral and dorsal somatic muscle specification as well as heart development, while Mef2 functions as a muscle differentiation factor (Azpiazu and Frasch, 1993; Bour et al., 1995). Due to its masterregulatory function, Twi has been extensively studied to elucidate its role in mesoderm determination and specification. Importantly, overexpression experiments revealed that Twi expression needs to be tightly regulated to prevent tissue malformation as well as loss and mis-location of cells (Baylies and Bate, 1996). In line, Twi expression is suppressed after 
mesoderm specification and is only maintained in adult muscle precursors (AMPs), a cell type that is silenced until remodelling of the muscle structure during metamorphosis (Bate et al., 1991). Thus, normal muscle differentiation requires the silencing of twi expression, however so far, the factors performing this critical function were unknown.

Here, we identified the Hox TF Ubx to inactivate the mesodermal master regulator Twi before muscle differentiation. Mesoderm-specific interference with Ubx maintained twi expression in many mesodermal cells and not just the AMPs, while premature activation of Ubx in the early mesoderm resulted in reduced twi expression. Importantly, we found that Ubx binds the twi promoter region together with the NK-homeodomain TF Tin and the MADS-Box TF Mef2, however required only Tin for this interaction. In addition, our results demonstrated that Tin and Mef2 are displaced from the promoter upon Ubx binding, which induced silencing of this region in cooperation with the Polycomb protein Pho. In sum, our study revealed that the Hox TF Ubx ensures normal muscle differentiation by repressing the mesodermal master regulator Twi. 


\section{Results}

\section{Ubx controls the expression of mesoderm specification and identity genes}

We and others previously uncovered the Hox TF Ubx to activate mesodermal genes (Kremser et al., 1999; Manak et al., 1995) and to repress the expression of alternative fate genes, which we showed to be crucial for the stabilization of the mesodermal tissue lineage (Domsch et al., 2019). Re-analysing the transcriptome dataset uncovered that the Ubxdependent control of tissue development is more complex than anticipated, as a substantial number of repressed Ubx targets were genes critical for mesoderm specification and differentiation. Based on our previous finding, we speculated that Ubx could control the repression of mesodermal identity genes by interacting with the Polycomb component Pleiohomeotic (Pho) to set repressive chromatin marks (Domsch et al., 2019). To test this assumption, we re-visited our established datasets (Domsch et al., 2019) and identified 504 chromatin regions bound by $\mathrm{Ubx}$ in the mesoderm that overlapped with active (H3K27ac) histone marks during mesoderm specification (stages 10-13, 4-9 hours after egg lay (AEL)), and switched to repressive marks (H3K27me3) during mesoderm differentiation (stages 1417, 10-18 hours AEL) (Fig. 1B). As $88 \%$ of these genomic regions were also bound by Pho (Fig. 1C), these results indicated that these regions experience a Pho-dependent switch to inactivation. Gene Ontology (GO) analysis of the genes associated with these genomic regions identified a significant over-representation of terms linked to stem cell commitment, early mesoderm development and gastrulation (Fig.1B). Intriguingly, we found that histone marks and changes correlated with genomic location, as the majority of Ubx binding events that changed their epigenetic status were found at promoter regions (Fig. 1D,E, SupFig 1A). And again, genes associated with such promoter regions controlled early specification events of the mesoderm (mesoderm cell fate specification, stem cell commitment etc) and encoded preferentially TFs of different classes (Fig. 1D,E,F, SupFig 1A). Enhancer regions were associated primarily with late muscle functions and structure genes (Fig. 1D,E, SupFig 1A).

The switch from active to repressive histone marks at promoter regions suggested that the associated genes get repressed in the course of mesoderm development. In order to provide direct evidence for this assumption, we tested whether the genes associated with these regions get de-repressed in the absence of Ubx. To this end, we overlapped the promoterassociated genes with genes previously identified as up-regulated when Ubx was mesoderm specifically degraded (Domsch et al., 2019). This analysis identified 32 genes, which fulfilled these requirements, including the early mesodermal master control gene twist (twi) (Fig. 1A). Importantly, twi expression, which persists only for a few hours throughout the mesoderm, gets induced very early during cellularisation to drive gastrulation and specification of mesodermal cell lineages (Leptin, 1991), while twi transcription is restricted to adult muscle precursors (AMPs) already in mid-embryogenesis (Bate et al., 1991). 
In sum, these data showed that Ubx repressed the expression of mesoderm identity genes including twi and suggested that Ubx performed this function by controlling the establishment of repressive chromatin marks.

\section{Ubx is required for twi repression at the onset of mesoderm differentiation}

In a next step, we investigated the requirements of Ubx in the mesodermal lineage in more detail. To efficiently eliminate Ubx activity in the mesoderm, we interfered with Ubx function using an alternative strategy to the protein degradation-based approach we had previously established, which strongly but not completely removed Ubx protein levels (Domsch et al., 2019). To this end, we targeted the Ubx gene using the inducible CRISPR/Cas9 mediated gene disruption, which relies on the tRNA-flanked multiplexed single guide RNA (gRNA) approach designed by Port and Bullock (Port et al., 2014; Port and Bullock, 2016) and the UAS/Gal4 system (Brand and Perrimon, 1993). Specifically, we generated a transgenic UAS line, which carries four Ubx specific gRNAs that bind within and close to the first Ubx exon, and combined it with the UAS-Cas9.P2 transgene (SupFig. 2A). In addition, we also generated a CRISPR line for another Hox gene, Antennapedia (Antp), using the same strategy as for Ubx to validate the specificity of our gene targeting approach (SupFig. 2A).

First, we validated the efficiency of gene disruption by ubiquitously expressing Ubx or Antp gRNAs and Cas9 using the armadillo (arm)-GAL4 driver (Sanson et al., 1996). qPCR analysis of total RNA isolated from embryos of late developmental stages (stage 14-17) revealed that the gRNAs function gene specifically, as a significant reduction of Antp or Ubx transcript levels was only observed when the respective gRNAs were expressed (SupFig. 2B). Importantly, Antp transcript levels were increased when the Ubx gene was disrupted (SupFig. 2B), which is in accordance with the known cross-regulatory interaction of Hox genes (Morata and Kerridge, 1982; Struhl, 1982). Indeed, it has been shown before that Antp expression is expanded in Ubx mutants, leading to a change of the identity of abdominal segments $A 1$ and A2 into the one of thoracic segments (Lewis, 1978).

After having confirmed the functionality and specificity of the UbxCRISPR system, we characterized the effects of interfering with Ubx specifically in the mesoderm in detail. Previously, we had used the Mef2-GAL4 driver, which starts to drive expression in embryonic stage 7 shortly before Ubx is active (Ranganayakulu et al., 1998). To interfere with the Ubx gene function already early in development, we combined the twist-GAL4 (2xPE twi-Gal4) driver, which starts to be active at embryonic stage 5 (Baker and Schubiger, 1996), with the Mef2-GAL4 driver. We investigated the effects of UbxCRISPR using this driver combination, which we refer to as twi\&Mef2-GAL4, and performed immunostaining for Ubx and the muscle structure protein Tropomyosin 1 (Tm1). In comparison to control animals, Ubx expression in muscles was significantly reduced in twi\&Mef2>UbxCRISPR embryos (Fig. 2A, $\mathrm{A}^{\prime}, \mathrm{B}, \mathrm{B}^{\prime}, \mathrm{E}$ ), while the neuronal expression was unaffected (Fig. 2C,D,E). In addition, the well-characterized 
Ubx expression in the visceral mesoderm, which marks the parasegment 7 (PS7), was absent in twi\&Mef2>UbxCRISPR embryos (Fig. 2C,D, yellow arrow head). Loss of Ubx expression was accompanied with defects in morphology, as muscles were malformed, detached or missing in UbxCRISPR embryos, which was not the case in control embryos (Fig. 2A,A",B,B", highlighted with an yellow arrow head). This muscle pattern resembled in part the phenotypes obtained when maintaining twi expression using Mef2-Gal4, in particular longer muscles that are significantly thinner towards their ends when compared to wild-type embryos (Fig. $2 A, A ", B, B "$, SupFig. 2E-G). As twi was among the Ubx target genes that remained expressed in the absence of Ubx (Fig. 1, SupFig. 1), we hypothesized that one of the functions of Ubx during mesoderm development was the timed repression of master control genes like twi at the onset of mesoderm differentiation. To provide evidence for this, we performed in situ hybridisations to monitor twi expression in mesoderm-specific Ubx loss of function (LOF) embryos (mesodermal UbxCRISPR). Investigation of stage 14 embryos revealed that twi transcript levels were significantly increased in abdominal segments of Ubx LOF embryos when compared to control animals (Fig. 2F,G,H highlighted with the yellow arrows), while thoracic twi expression was unaffected (Fig. 2H). Conversely, when we induced Ubx expression already early during mesoderm specification using the twi-GAL4 driver, twi transcript levels were reduced in particular in thoracic segments of stage 11 (but also of later stage) embryos (Fig. 2I,J,K, highlighted with the yellow arrows).

In sum, these results highlighted that Ubx function was required and sufficient for twi transcriptional repression.

\section{Complex interplay of Ubx, Tin and Mef2 at the twi promoter}

It has been shown previously that twi expression starts during cellularisation under the control of dl (Ray et al., 1991; Thisse et al., 1991) and maintains its own expression during mesoderm specification (Levine and Davidson, 2005; Sandmann et al., 2007). In addition, other TFs control twi expression (Sandmann et al., 2007), including Myocyte enhancer factor 2 (Mef2), which is generally required for muscle development and expressed in embryonic and larval muscles (Bour et al., 1995), and Tinman (Tin), which is restricted to the embryonic heart cell precursors by stage 12 (Azpiazu and Frasch, 1993) (Fig.3A). Importantly, Mef2 and Tin positively control twi transcription by interacting with the twi promoter (Sandmann et al., 2007), a region we found to be bound by Ubx (Fig 1A) (Domsch et al., 2019). This raised the intriguing possibility that $\mathrm{Ubx}$ replaces one or both of these TFs at the twi promoter thereby initiating twi repression at the onset of differentiation. To test this hypothesis, we studied the relationship of Ubx, Tin and Mef2 at the twi promoter. We performed ChIP-qPCR experiments for all three factors at three different time points, representing the determination and early specification (stages 7 to 9), the specification (stages 10 to 12) and the differentiation (stages 14 to 17) stages of the mesoderm (Fig. 3A). To precisely map TF interaction sites, we divided the twi 
promoter into four regions (Fig. 3A). We found Tin and Mef2 to strongly interact with all regions of the twi promoter at embryonic stages 7 to 9 , while binding was significantly decreased during stages 10 to 12, which became even more pronounced during stages 14 to 17 (Fig.3B). The converse binding behaviour was observed for Ubx. Consistent with the late onset of Ubx expression, we did not detect Ubx interactions with the twi promoter at stages 7 to 9 , while we found Ubx to specifically bind region 1 and 5 starting at stages 10 to 12 , which was strongly increased at stages 14 to 17 (Fig. 3B). Intriguingly, region 5 experienced the most profound reduction in Tin and Mef2 binding as soon as Ubx started to interact with this region (Fig. 3B), suggesting that Ubx caused the clearance of Tin and Mef2 protein from the twi promoter. To test this possibility, we performed Tin and Mef2 ChIP-qPCR experiments in mesodermal cells devoid lacking Ubx gene expression (UbxCRISPR). We chose stage 10 to 12 embryos for this analysis, as this time frame represents the transition phase when twi expression starts to be repressed. Intriguingly, binding of both TFs was significantly increased at regions 4, 1 and 5 in the absence of Ubx (Fig.3C). As expression of Mef2 and Tin was unchanged in Ubx mutants (SupFig.3C,D,K), these results showed that Ubx plays an important role in preventing binding of these two TFs to the twi promoter.

In a next step, we wanted to correlate TF interactions at the twi promoter with expression. Thus, we quantified twi transcript levels in abdominal segments of Ubx, tin as well as Mef2 mutants. We found twi transcription to be unaffected in stage $11 \mathrm{Ubx}$, Mef2 and tin heterozygous mutant embryos (Fig. 3D,E,F,L). Indeed, it resembled the characteristic twi expression in wild-type embryos with reduced twi transcript levels in abdominal compared to thoracic segments (SupFig. 2C,D). In contrast, twi transcription was increased in abdominal segments of Ubx and tin homozygous mutants (Fig.3G,I,L, highlighted by the yellow arrows), while expression was severely reduced in Mef2 homozygous mutants (Fig.3H,L, highlighted by the yellow arrows). In addition, the expression of Ubx, Tin and Mef2 was unaffected in the respective mutant backgrounds (SupFig.3 A-D, I-K). This result indicated that Mef2 plays a critical role in maintaining the twi expression even at the onset of differentiation and not only during mesoderm specification. Furthermore, the correlation of increased Mef2 binding to the twi promoter (Fig. 3C) and increased twi transcription in the absence of Ubx (Fig. 3D,G,L) strengthened our hypothesis that Ubx replaces Mef2 at the twi promoter at the onset of mesoderm differentiation to inhibit twi activation. The interplay of Ubx and Tin is more complex, as binding of Tin to the twi promoter was also increased in the absence of Ubx (Fig. 3C). As for Mef2, this is indicative of a maintenance function of Tin, as twi transcription is increased in Ubx mutants (Fig. 2G,L). However, in contrast to Mef2 removal of Tin resulted in increased and not decreased twi expression levels (Fig. 3I,L), suggesting that Tin is directly or indirectly involved in the Ubx-mediated twi repression. As Ubx is expressed at similar levels in the mesoderm in tin mutants (SupFig. $3 \mathrm{~A}, \mathrm{I}$ ), we assumed that Ubx requires Tin for the initial 
interaction with region 5 of the twi promoter, and not for later functions. In order to provide additional evidence, we investigated twi expression levels in heterozygous Mef2, Ubx as well as tin, Ubx double mutants. We found twi expression levels in heterozygous Mef2, Ubx double mutants to be comparable to single heterozygous conditions (Fig. 3J,L), whereas in tin, Ubx heterozygous conditions a significant increase of twi expression was noticeable.

These results indicated that Ubx interacts with Tin but not with Mef2 to initiate the repression of twi.

\section{Ubx requires Tin to interact with the twi promoter}

To further investigate the requirements of $U b x$ in twi repression, we overexpressed $U b x$ in Mef2 or tin homozygous mutants using the twi-GAL4 driver and characterized twi transcript (and Ubx protein) expression in stage 10 embryos when Ubx is normally not active. Homozygous tin and Mef2 mutant embryos did not display an obvious phenotype at this stage, as twi positive myoblasts were visible as a thin layer above the ectoderm (Fig.4A,C). In contrast, overexpression of Ubx in Mef2 mutants resulted in a severe reduction of twi expression (Fig.4B), while Ubx overexpression in tin mutants did not affect twi expression (Fig.4D). These results demonstrated that Ubx required Tin function for twi repression.

In a next step, we analysed the interdependency of Ubx and Tin in more detail by testing the binding of Ubx to the twi promoter in the absence of Tin. To this end, we abolished Tin function in them mesoderm by using the inducible CRISPR/Cas9 mediated gene disruption to target the tin gene (SupFig. 3E-H). Mesoderm-specific expression of a tin specific gRNA (tinCRISPR) by means of the twi\&Mef2-GAL4 driver resulted in a significant reduction of Tin protein levels in twi\&Mef2>tinCRISPR embryos in comparison to control (twi\&Mef2-Gal4) embryos at stage 11 (SupFig. $3 \mathrm{~F}-\mathrm{H}$ ), validating efficient tin gene disruption. Subsequently, we performed Ubx-ChIP analysis in tinCRISPR embryos focusing on the twi promoter regions 1 and 5 , which revealed a significant reduction of Ubx binding to these sites in the absence of Tin (Fig.4E). We had shown previously that Ubx repressed the expression of alternative fate genes by stabilising binding of the Polycomb protein Pho and the setting of repressive chromatin marks at control regions close to such genes (Domsch et al., 2019). Thus, we tested whether a similar mechanism applied to the repression of the master control gene twi, and investigated Pho binding, H3K27me3 and H3K27ac levels at the twi promoter in the absence of Tin and Ubx. Using ChIP-qPCR, we found Pho binding and H3K27me3 levels to be significantly reduced when the tin or the Ubx gene were disrupted, while H3K27ac levels were not changed in comparison to the control (Fig.4E, F). The results indicated that Ubx employs a similar mechanism as for the alternative fate gene repression and interacts with Pho at the twi promoter region. Further on, Ubx requires Tin also at the molecular level to interact with the twi regulatory element. 
bioRxiv preprint doi: https://doi.org/10.1101/2020.02.24.963231; this version posted February 25, 2020. The copyright holder for this preprint (which was not certified by peer review) is the author/funder. All rights reserved. No reuse allowed without permission. 


\section{Discussion}

Master regulators instruct cells to adopt specific fates by activating complex gene regulatory networks. One such master regulator is Twi, which is critically required for mesoderm determination and specification in Drosophila. In order to progress in development and to enable differentiation, early specification factors need to be silenced, which is also the case for Twi during mesoderm development. However so far, the factors performing this critical function are largely unknown. Our study now identified the Hox TF Ubx to be critical for the inactivation of the mesodermal master regulator Twi, thereby ensuring the transition from mesoderm specification to differentiation. Based on our data, we propose the following model (Fig.4G). During late stages in mesoderm determination (stages 7 to 9), the twi promoter is bound by Tin and Mef2 to maintain twi expression. At the beginning of specification (stages 10 to 12), both factors are replaced by Ubx, which recruits the Polycomb protein Pho and initiates repression of twi transcription by establishing repressive chromatin marks. Interaction of Ubx with the twi promoter for repression requires Tin, while Mef2 seems to ensure the proper timing of twi activation versus repression. Once twi repression is mediated under the control of Ubx and Pho, coordinated differentiation of specified myoblasts into functional muscle fibers can be initiated (stages 14-17).

How master-regulatory networks are changed for the transition into the next developmental stage has been studied in a few cases, which led to the formulation of two mechanisms: 1) a context-specific network rewiring, and/or 2) a switch of regulatory relationships to assemble new control hierarchies (Atkins et al., 2013; Davis and Rebay, 2017).

During Drosophila eye development, a positive feedback loop essential to maintain Eyeless (Ey) expression is disrupted to proceed with photoreceptor fate specification and neuronal differentiation (Atkins et al., 2013). The inactivation of ey is achieved by unwiring the network and promoting the interaction of Eyes absent (Eya) and Sine oculis (So), which leads to direct repression of ey in differentiating cells and breaks the Ey regulatory network (Atkins et al., 2013). In our case, the Twi network is essential for mesoderm determination and specification, since Twi activates essential lineage identity genes that help to maintain its activation (Sandmann et al., 2007). The need for a tight regulation of twi expression is indicated by overexpression experiments showing severe tissue malformations (Baylies and Bate, 1996) and muscle abnormalities by maintaining Twi activity (SupFig.2). We assume the binding of Ubx to the twi promoter to mark the transition from specification to differentiation by downregulating twi and inducing a break/unwiring of the Twi network (Fig4). Repression of twi uncouples the Tin and Mef2 networks, which maintain an independent activity within the developing heart precursors as well as in myoblasts that will differentiate into functional muscle fibers, respectively (Azpiazu and Frasch, 1993; Bour et al., 1995; Sandmann et al., 2007). 
Hox TFs are themselves master regulators of segment identity (Pearson et al., 2005). For example, loss of Ubx leads to a well-known homeotic transformation of the abdominal into thoracic segments promoted by Antennapedia (Antp), which shows that these TFs initiate their own regulatory network (Lewis, 1978). In the mesoderm, the inactivation of twi may lead to the activation of the Ubx control network. This network will in turn promote muscle differentiation and stable lineage commitment through the activation of genes for the progression of tissue development, like decapentaplegic (dpp) and betaTubulin 60D (betaTub60D) in the visceral mesoderm (Kremser et al., 1999; Manak et al., 1995). Importantly, we assume differentiation process to not inhibited but negatively influenced, since maintaining the twi expression during muscle differentiation leads to malformations but not severe muscle pattern or structure defects. Twi itself activates the muscle differentiation factor Mef2. Intriguingly, it is known that increased amounts of Mef2 proteins have a drastic impact on normal muscle formations and structures (Huang, 2000; Molkentin and Olson, 1996). This indicates that the phenotype observed in mild twi overexpression or loss of Ubx function experiments is caused by increased expression of Mef2, resulting in an imbalance in protein amounts (Huang, 2000; Molkentin and Olson, 1996).

To switch or unwire the Twi network, Ubx has to interact with the regulatory regions of twi. Our data, which show that Pho is unable to bind to the twi promoter in the absence of Ubx, suggest that Ubx binds to the twi promoter and recruits Pho to initiate twi inactivation via the assembly of the Polycomb complex. In addition, we find that $\mathrm{Ubx}$ is unable to interact with this region without Tin, suggesting that this TF "prepares" the chromatin for Ubx binding. This is in line with previous studies showing Tin serves as a chromatin anchoring protein in ventral myoblasts (Busser et al., 2013; Liu et al., 2009).

The function of Mef2 in twi repression is less clear, since the expression of twi is unaffected in Mef2 mutants. However, our data also suggest that Mef2 plays a role, as the Ubx mediated repression of $t w i$ in the abdominal segments occurs earlier in the absence of Mef2. Thus, we assume Mef2 to control the timing of specification and differentiation. It has been shown before that Mef2 protein binds to the minor groove and bends the DNA in cooperation with co-factors (Huang, 2000). Taken together, we assume the Twi regulatory feedback loop is unwired through the binding of Ubx to the twi promoter region (Fig.4J).

In future, it will be important to also focus on the inactivation of essential master regulators and how the repression complex is established and formed, to understand in more detail how cells, which are devoted for differentiation, enter their developmental path. 


\section{Materials and Methods}

\section{Fly stocks and husbandry}

For CRISPR experiments, the following lines were used: UAS-Cas9.P2 obtained from Filip Port, Mef2-GAL4 (BL50742), twi-(2xPE)-GAL4 (BL2517), arm-GAL4 (BL1561), w $w^{1118}$ (BL3605), Mef2 ${ }^{22-21} / \mathrm{CyO}$ wg-lacZ given by Hanh Nguyen, $\mathrm{tin}^{346} / \mathrm{TM} 3 \mathrm{ftz-lacZ}$ gift from Manfred Frasch and Ubx ${ }^{1} / T M 6$ Dfd-LacZ from Ana Rogulia-Ortmann, UAS-gRNA-Ubx, UAS-gRNAAntp, UAS-gRNA-Tin and UAS-HA-Ubx overexpression constructs were made during the study. The mutants and overexpression constructs were crossed together accordingly for genetic interaction experiments.

\section{Generation of Ubx, Antp \& tin UAS-gRNA and UAS-HA-Ubx constructs}

For the UAS-gRNAs: The gRNAs were design using the technique and tool published by Filip Port implying tRNAs separated target-specific gRNAs (F. Port et al., 2014; Port and Bullock, 2016)in combination with the UAS/GAL4 system (Brand and Perrimon, 1993). Suitable gRNAs were identified using the webtool CRISPR Optimal Traget Finder (http://targetfinder.flycrispr.neuro.brown.edu) and the first exon as well as including flanking regions intronic regions for Ubx and Antp. The $20 \mathrm{nt}$ were amplified and cloned in the pCFD6 plasmid according to the protocol published on https://www.crisprflydesign.org (Ubx-gRNA1: GGAGGCGTACAGAGCGGCGT， Ubx-gRNA2: GGCGAGCGGTAAAGCGCTGA， UbxgRNA3: GGGCGCCGCTGCCCAAACGG, Ubx-gRNA4: AAGAGTAGGTCAGCCGAAGG, Antp-gRNA1: GATGACGCTGCCCCATCACA, Antp-gRNA2: GGCCGTTGTAGTAGGGCATG, Antp-grNA3: GGCGGGATCAGCAGACGCTG, AntpgRNA4: GGTTCTGATGGACCTGTGAT, Tin-gRNA1: GAGCATCTACGGTTCAGATG, TingRNA2: GGACCTCAACAGCTCCGCTG, Tin-gRNA3: GCTTCTGCGTCGGAGCTTGC, TingRNA4: GAGGGCGTGACGTATGGCGT).

For the overexpression construct: The full Ubx coding region (Ubx isoform I) was cloned into the pUAS-attB vector (Bischof et al., 2007) using a forward primer containing a EcoRI restriction site and the $\mathrm{HA}$ tag sequence as well as a revers primer with a Xbal restriction site. The UAS-constructs were injected by BestGene on attP5 (second chromosome, gRNA, UASUbx) and attP2 (third chromosome, gRNA) carrying fly lines. Primers and sequence maps are available upon request.

\section{ChIP experiments and isolation of total RNA}

ChIP experiments were performed as described in Sandmann et al., 2007 and analyzed by using qPCR experiments (Invitrogen Syber-Green-Mix, Primers are available upon request). The following antibodies were used: H3K27ac (ab4729, Abcam), H3K27me3 (ab6002, Abcam), gp-Ubx (Domsch et al., 2019), Rb-Mef2 (gift from Hanh Nguyen) and Rb-Tin (gift from Manfred Frasch (Jin et al., 2013). ChIP-qPCR results were calculated as described in Schaub et al., 2012. Total RNA was isolated with TRIZol (Invitrogen), used to generate cDNA 
(TermoFisher Scientific, RevertAid First Strand cDNA Synthesis Kit) and the material was analysed or validated by qPCR (Invitrogen Syber-Green-Mix, ChIP-Primers: twi promoter region 1 (twi_1): for GCCGACAATTCCCCTCGTAT, rev GGCGGGGGATTTCGATTTTC; twi promoter region 3 (twi_3): $\quad$ TTTTCGTGGAGTTCCCCTCG, TGGGATCGCAGGAATTTCCC; twi promoter region 4 (twi_4): for CGCTGCAAACAACAACATTCA, rev TCTTTGGATCGACGGGAATTCA; twi promoter region 5 (twi_5): for CGTCGAGTCAAGGCTCTCTT, rev CATCCCGCTCCCACTCAATG; scramb1 (negative control): for GAGCGAAAAGGTGCAGAAAG, rev CCTGTCTGTTTCCCTGCTGT; region on the $2^{\text {nd }}$ chromosome left arm $2 L$ (negative control): for AGgTGTTGTTGTGGGTCCTT, rev TCCCAGAGTTCCCTTTAGCA; RNA-Primers: UbxPrimer: for AGTTTACACCAGGCCAG CAA, rev GCCCGCAAGAGATTCTGAGT; Antp-Primer: for GGGCTATACGGACGTTGG AG, rev ATGTCCCTGGTGCATCTGTG; RpL32-Primer (Steiner et al., 2012): for TACAGGCCCAAGATCGTG AA, rev TCTCCTTGCGCTTCTTGGA). Bioinformatics analysis

Bioinformatics analysis was performed as described in (Domsch et al., 2019). Material and files used for the analysis were generated and published in (Domsch et al., 2019) (storage: NCBI Gene Expression Omnibus GSE121754, GSE121670, GSE12175). Visualization of the data was performed as described in (Domsch et al., 2019).

\section{Immunofluorescence staining and microscopy}

Embryonic stainings were performed as described in (Domsch et al., 2019) and the following antibodies were used: Rat-Tm1 (1:200, MAC-141, ab50567, Abcam), gp-Ubx (Domsch et al., 2019), Rb-Tin (1:1000, gift from Manfred Frasch, (Jin et al., 2013)) and gp-Twi (1:500, gift from Eileen Furlong). For in-situ hybridizations a 1000 bp fragment of the twi was amplified and served as a probe template. The generation of the probe and the in-situ hybridization was performed as described in (Schindelin et al., 2012). Amplification was obtained with the TSA system (Perkin-Elmer). Biotinylated (1:200, Vector Labs) and fluorescent (1:200, Jackson ImmunoResearch or Invitrogen) secondary antibodies were used. Images were acquired on the Leica SP8 Microscope (20x/0.7 HC PL APO Glycerol, 63x/1.3 HC PL APO Glycerol). The collected images were analyzed and processed with Leica Application Suite X (LAS X, Leica Microsystems) and Adobe Photoshop.

\section{Quantifications of data}

Quantification of the GFP signal intensity: GFP signal was measured with the FIJI/ImageJ (v1.52p) (Schindelin et al., 2012) from at least 5 segments per embryo in 5 embryos. The mean and the t-test for significance were calculated in Microsoft Excel and graphic illustrations were performed in R (R Core Team, 2018). 


\section{Acknowledgments}

We are grateful and like to thank the fly community and especially Filip Port for CRISPR fly stocks, Hanh Nguyen, Manfred Frasch, Ana Rogulia-Ortmann and Eileen Furlong for antibodies as well as BestGene and the Lohmann lab for critical comments on the manuscript. The project was founded by the DFG grand LO 844 8/1 to Ingrid Lohmann.

\section{Competing interests:}

The authors declare no competing or financial interests.

\section{Authors contribution}

Conceptualization: K.D.

Methodology: K.D., J.S., M.J., C.S.

Validation: K.D., J.S.

Investigation: K.D., J.S., M.J.

Data curation: K.D.

Visualisation: K.D., J.S.

Supervision: K.D.

Project administration: K.D., I.L.

Funding acquisition: I.L.

Writing - original draft: K.D., I.L.

Writing - review \& editing: K.D., I.L. 


\section{Figure Legends}

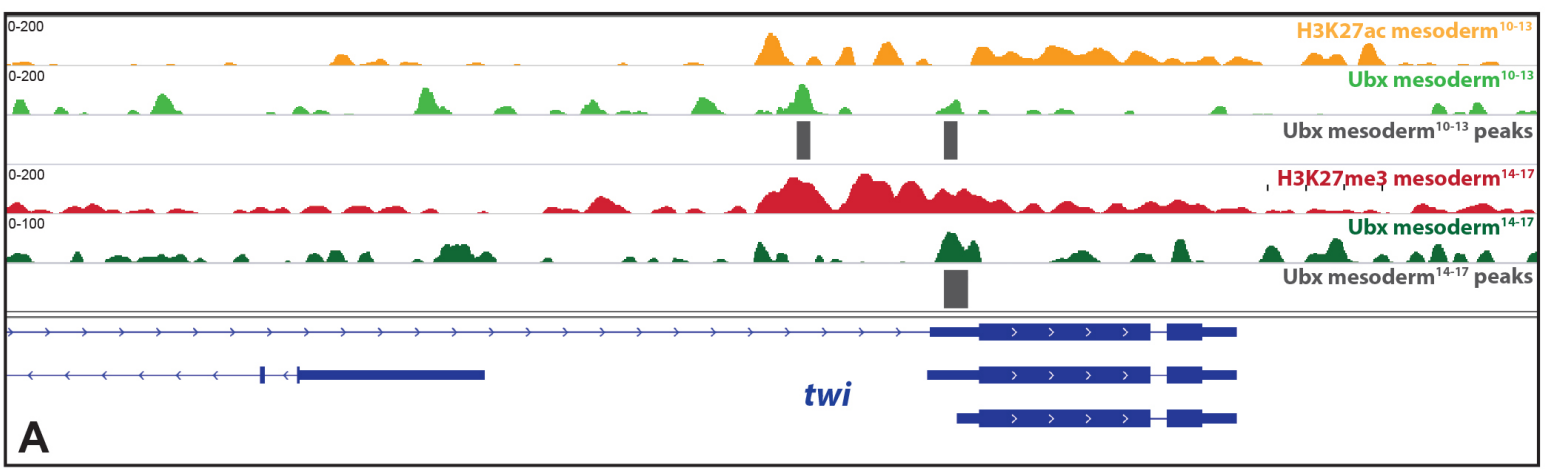

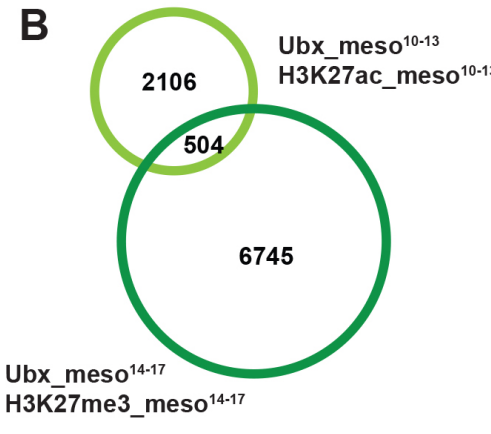

D

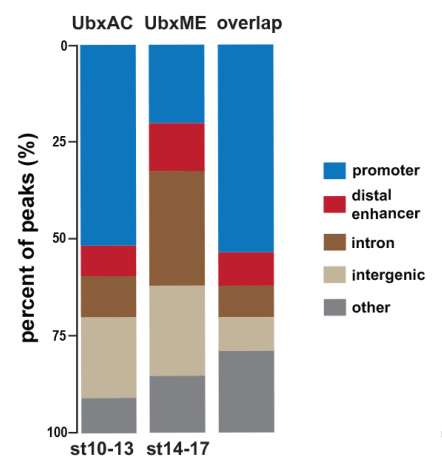

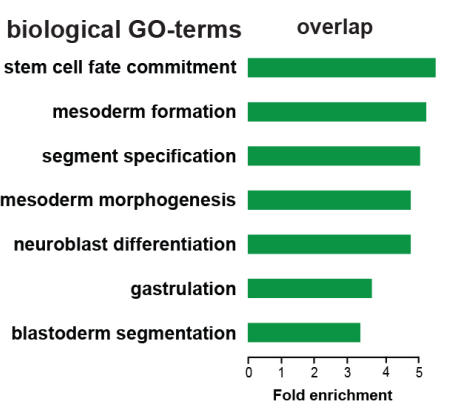

E

molecular GO-terms enhancer binding

transcription corepressor activity

Polymerase II regulatory region DNA binding

transcription regulatory region sequence

-specific DNA binding

peptide hormone binding

collagen binding

DNA-binding transcription

protein binding

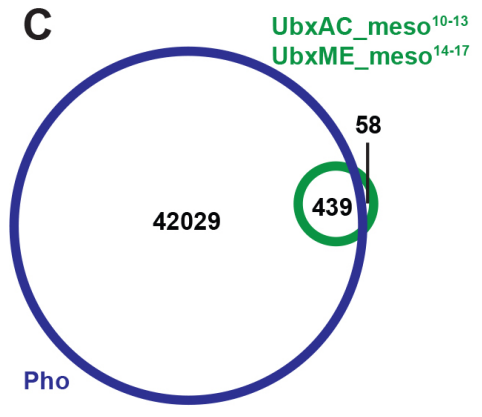

$\mathbf{F}$

protein classes - promoter DNA-binding

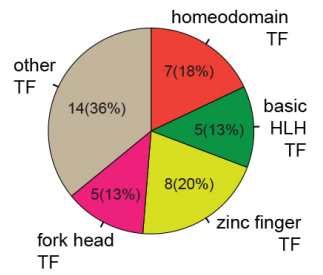

TF

Figure 1: Ubx genomic interactions during mesoderm development.

A) Illustration of the twi genomic region, showing Ubx binding and histone mark distribution at different developmental stages. Yellow: acetylation H3K27ac stages 10-13, red: methylation H3K27me3 stages 14-17, light green: Ubx stages 10-13, dark green: Ubx stages 14-17, grey boxes: accepted Ubx peaks, blue: coding region of the gene. B) Overlap of Ubx binding events associated with acetylated or methylated histone marks at different stages in the mesoderm, biological GO-term analysis (green) of the overlap. C) Overlap of Pho binding events and the genes co-bound by Ubx in different stages identified in B. D) Comparison of the localization of peaks called within unique genomic regions of Ubx. Localization are classified as promoters (1000-+10 bp from TSS, 5' UTR), distal enhancers (-2000 to -1000 from TSS, 3' UTR, downstream), intron (intronic regions), intergenic (distal intergenic) and other regions (including exons). E) Molecular GO-term analysis of the overlap divided in enhancer (red) and promoter (blue) regions. F) Pie chart of the identified protein classes of genes that are bound by Ubx at the promoter region and have a function associated with DNA-binding. 
A

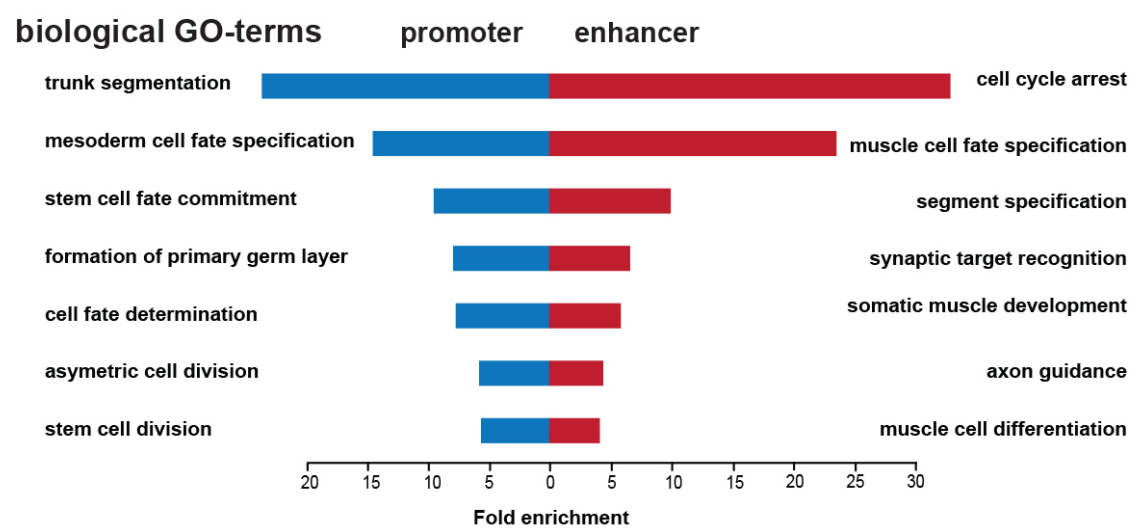

B

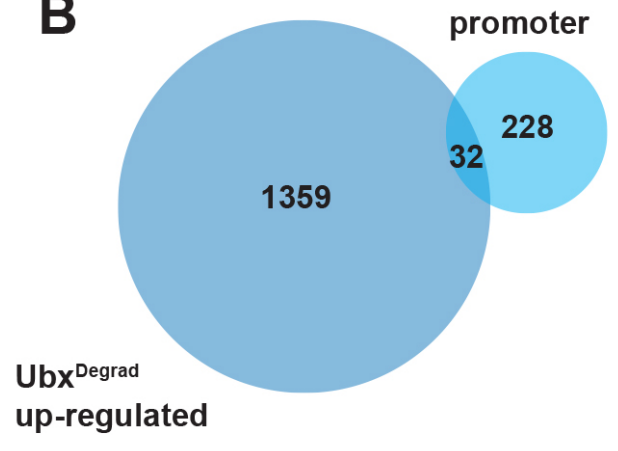

molecular GO-terms overlap

Wnt-protein binding

G protein-coupled receptor binding

protein homodimerization activity

signaling receptor activity

DNA-binding transcription factor activity

transcription regulation activity

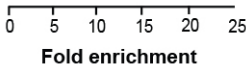

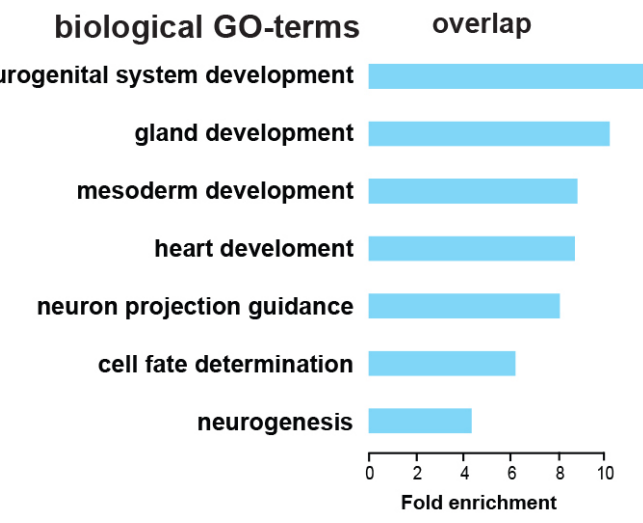

\section{SupFigure 1: Ubx genomic interactions during mesoderm development.}

A) Biological GO-term analysis of the overlap from Fig.1B divided in enhancer (red) and promoter (blue) regions. B) Overlap of genes that are up-regulated in the UbxDegrad (dark blue) background and promoter regions from the Ubx overlap shown in Fig.1B (light blue), biological and molecular GO-term analysis of the 32 genes from the overlap in B. 

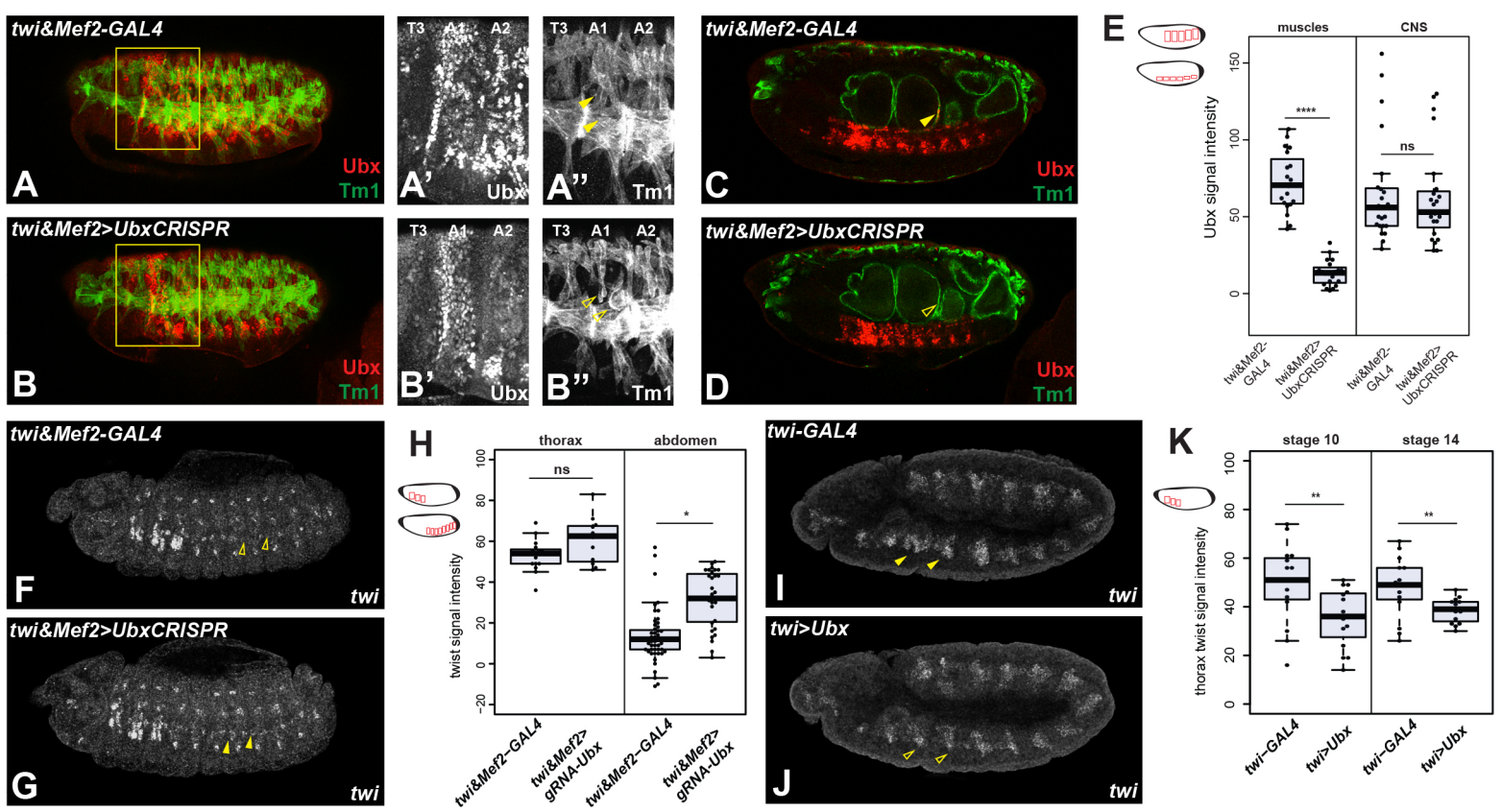

Figure 2: Ubx represses twi transcription.

A-D) Control (twi\&Mef2-Gal4) and UbxCRISPR (twi\&Mef2>UbxCRISPR) stage 16 embryos stained with Tropomyosin (Tm1, green) and Ubx (red). Box highlights third thoracic and first, second abdominal segments. A', B') enlargement in grey scale, thoracic segment 3 (T3) and abdominal segments $1 \& 2$ ( $11, A 2)$, showing loss of Ubx expression in the mesoderm while expression in the ectoderm is unaffected. A",B") enlargement in grey scale of Tm1, open yellow arrowheads indicate the missing muscles in UbxCRISPR compared to the control (closed arrowheads). C,D) gut and CNS view of A and B, indicating the maintenance of Ubx expression in the CNS and missing visceral mesoderm expression in UbxCRISPR (empty arrow head) embryos. E) Validation of the Ubx expression in the muscle and CNS, illustration of the measured region in red, up: mesoderm, down: CNS, showing that the CNS expression is not affected by the UbxCRISPR but the mesodermal expression in decreased, ${ }^{* * \star *} p=3.02 \mathrm{e}-$ 15. $\mathbf{F , G}$ ) in situ hybridisation of control (twi\&Mef2-Ga/4) and UbxCRISPR (twi\&Mef2>UbxCRISPR) embryos stage 14 using twi probe, closed arrowheads indicate additional twi expression in the UbxCRISPR. $\mathrm{H}$ ) Validation of twi expression increase, illustration of the measured region in red, up: thorax, down: abdomen, showing that the thoracic expression is unchanged whereas the abdominal expression increased in UbxCRISPR experiments, ${ }^{*} p=0.03$, ns: non-significant. I,J) in situ hybridisation of control (twi-Ga/4) and mesodermal Ubx overexpression (twi>Ubx) embryos stage 10 using twi probe, displaying a reduced twi expression in the overexpression (closed yellow arrow) K) Validation of twi expression decrease, illustration of the measured region in red (thorax), stage $10:{ }^{* *} p=0.01$, stage $14:{ }^{* *} p=0.008$. 
bioRxiv preprint doi: https://doi.org/10.1101/2020.02.24.963231; this version posted February 25, 2020. The copyright holder for this preprint (which was not certified by peer review) is the author/funder. All rights reserved. No reuse allowed without permission. 

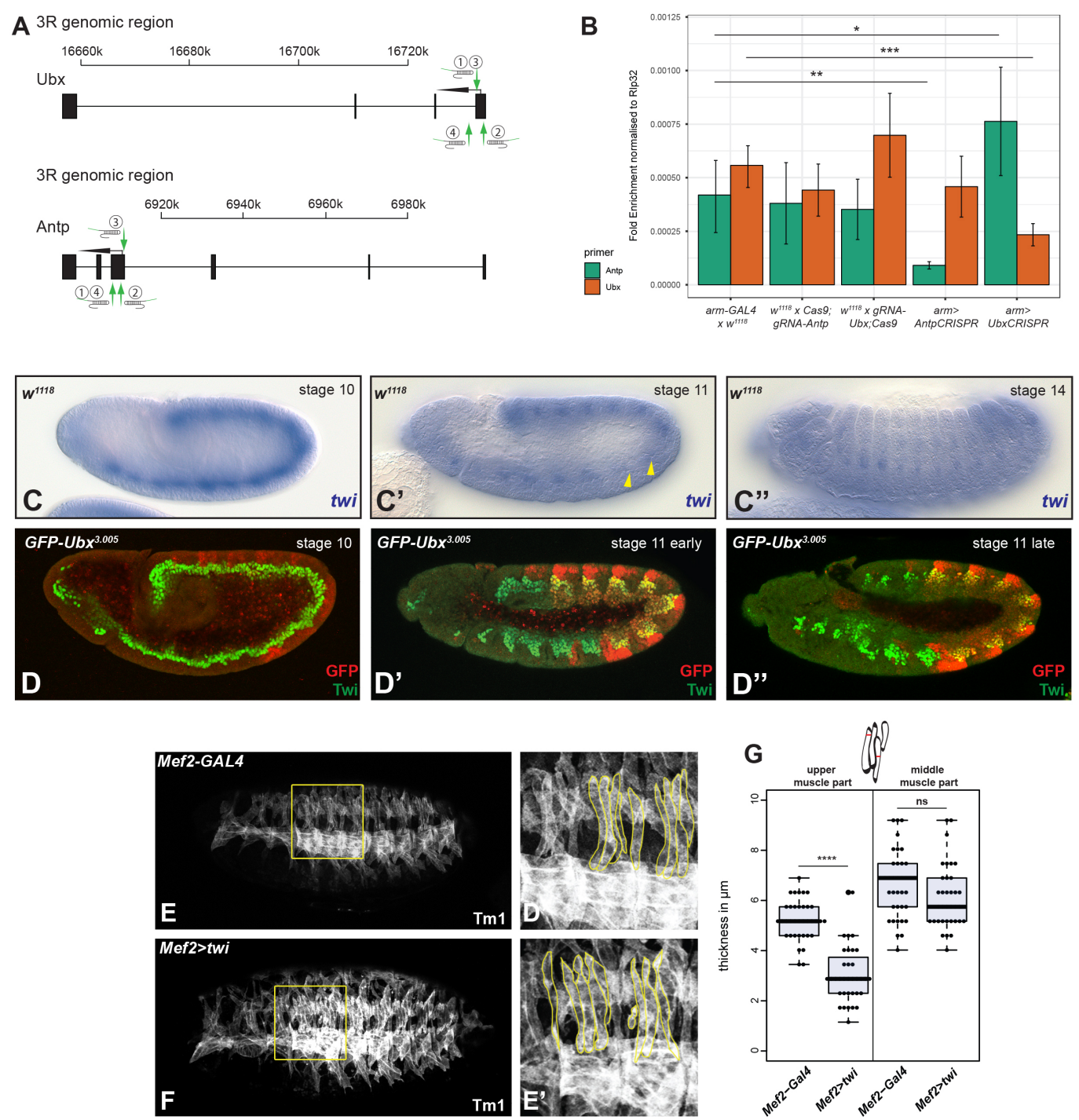

\section{SupFigure 2: Ubx represses the twi transcription.}

A) Localisation of the Ubx and Antp gRNA. B) Validation of the Ubx- (orange) and AntpCRISPR (green) using arm-Gal4. qPCR results show a downregulation of Antp transcripts in the AntpCRISPR and Ubx transcripts in the UbxCRISPR when compared to the controls. In addition, Antp transcripts are upregulated in the UbxCRISPR. Ubx in UbxCRISPR: *** $\mathrm{p}=0.00074$, Antp in AntpCRISPR ${ }^{* *} \mathrm{p}=0.0137$, Antp in UbxCRISPR: * $\mathrm{p}=0.0089$. C) insitu hybridisation of $w^{1118}$ embryos (wildtype) of different stages using twi probe, closed yellow arrow indicates the abdominal segments A1 und A2. D) Endogenous GFP-Ubx embryos stained with GFP (red, labelling GFP-Ubx) and Twi (green) at different stages. E,F) Control (Mef2-Gal4) and twi overexpression experiments (Mef2>twi) stage 16 embryos stained with Tropomyosin (Tm1), illustrating the lateral muscle pattern in. Box highlights third thoracic and first, second abdominal segments. E',F') Enlargement and highlighted (yellow) lateral transversal and segment boundary muscles. G) Validation of the muscle thickness lateral transversal muscles, illustration of the measured region above and measured region indicated 
bioRxiv preprint doi: https://doi.org/10.1101/2020.02.24.963231; this version posted February 25, 2020. The copyright holder for this preprint (which was not certified by peer review) is the author/funder. All rights reserved. No reuse allowed without permission.

in a red line, showing that the middle thickness is not affected but the end of the muscles show significant defects in thickness, ${ }^{* * * *} p=1.10 \mathrm{e}-10$, ns: non-significant. 
A
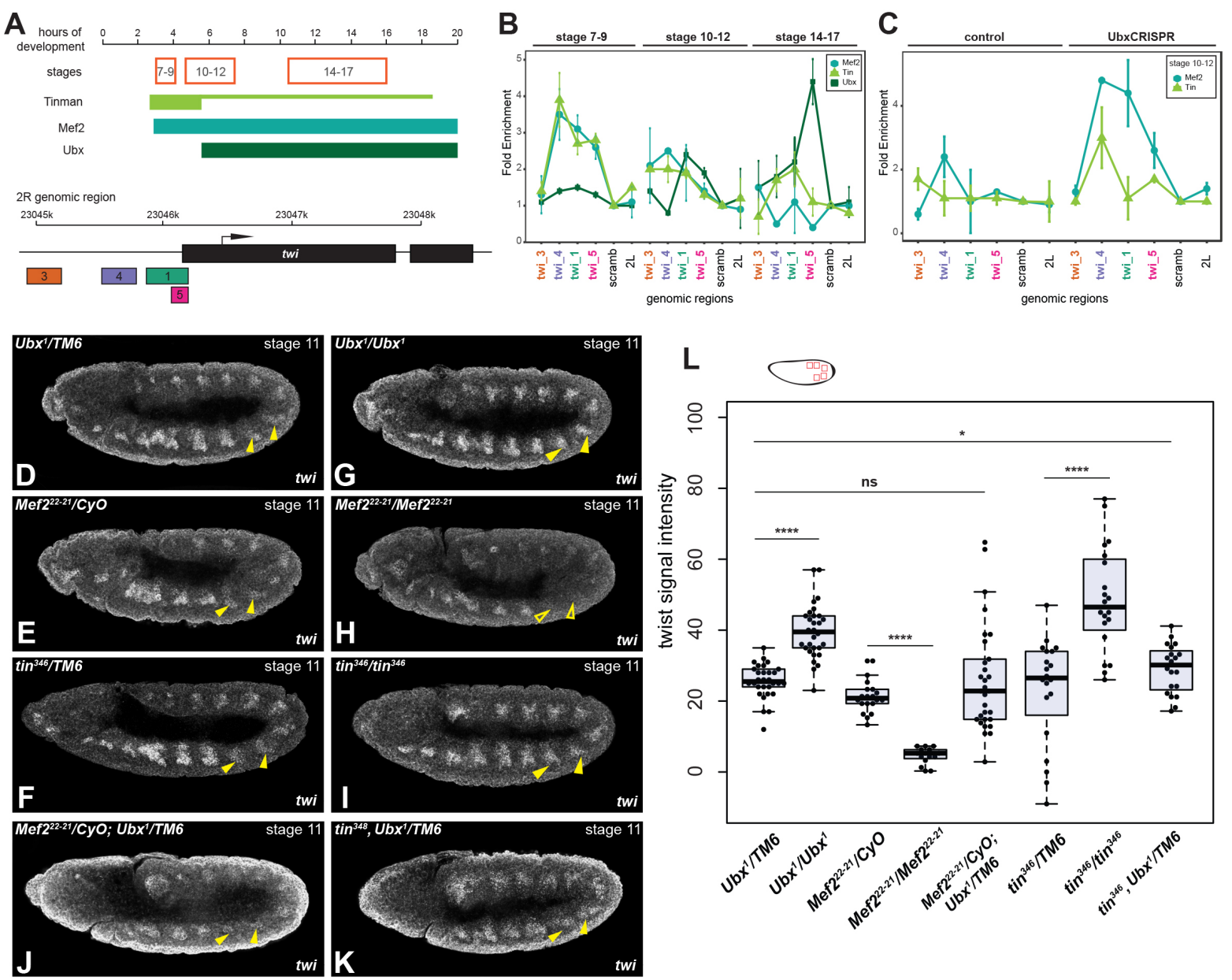

Figure 3: Ubx genetically interacts with Tin but not with Mef2.

A) Up panel: time scale with analysed stages (red box), illustration of Tin, Mef2 and Ubx expression regarding the hours of the development. Lower panel: twi genomic region, illustration of the analysed promoter regions in a respected colour code. B) Mef2, Tin and Ubx ChIP-qPCR experiments on wildtype chromatin at different stages, showing that Tin and Mef2 bind to the twi promoter during stages 7-9 and then their binding is reduced in the following, whereas $\mathrm{Ubx}$ is expressed at stages $10-12$ and starts to significantly accumulate at the promoter over time C) Mef2 and Tin ChIP-qPCR experiments using control (twi\&Mef2-Gal4) and UbxCRISPR (twi\&Mef2>UbxCRISPR) chromatin at stage 10-12, Mef2 and Tin binding is maintained in the Ubx know-down background. D-K) in situ hybridisation of Ubx, tin and Mef2 heterozygous $(\mathrm{D}, \mathrm{E}, \mathrm{F})$ and homozygous $(\mathrm{G}, \mathrm{H}, \mathrm{I})$ mutants stage 11 using twi probe, yellow arrow heads indicate the abdominal segments $1 \& 2$, showing an increase of twi expression in Ubx and tin homozygous mutants (closed arrow) and decrease in Mef2 mutants (open arrow), in situ hybridisation in double heterozygous background of Mef2, Ubx (J) and tin, Ubx (K) stage 11 show an increase of twi in tin, Ubx heterozygous conditions (closed arrow). L) Validation of twi expression, illustration of the measured region in red (abdomen), Ubx: ${ }^{* * *} p=1.03 e-11$, Mef2: ${ }^{* * * *} p=3.68 \mathrm{e}-14$, tin: ${ }^{* * *} p=3.56 \mathrm{e}-6$, tin, Ubx heterozygous: * $p=0.0467$, ns: nonsignificant. 
bioRxiv preprint doi: https://doi.org/10.1101/2020.02.24.963231; this version posted February 25, 2020. The copyright holder for this preprint (which was not certified by peer review) is the author/funder. All rights reserved. No reuse allowed without permission. 

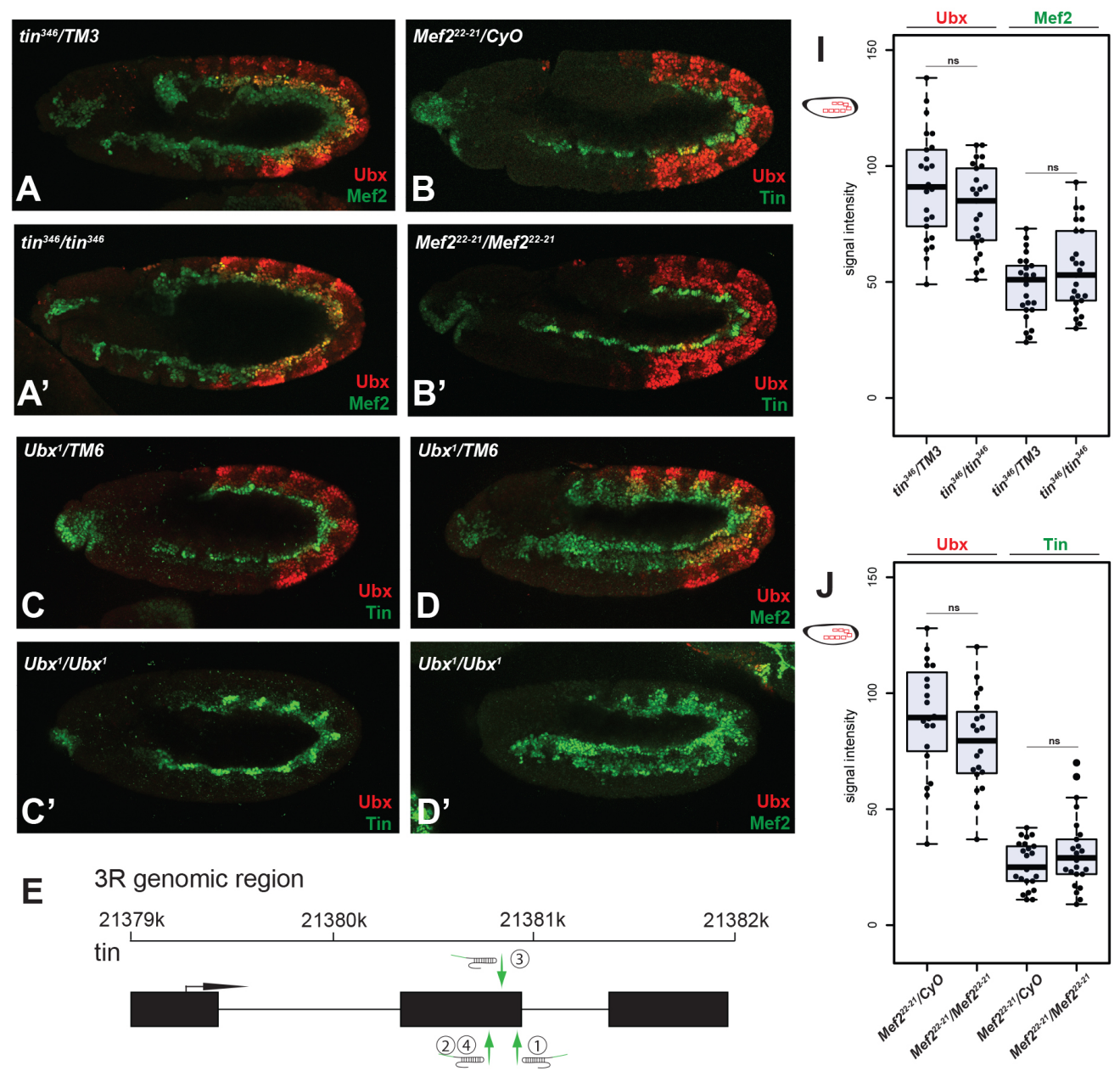

E
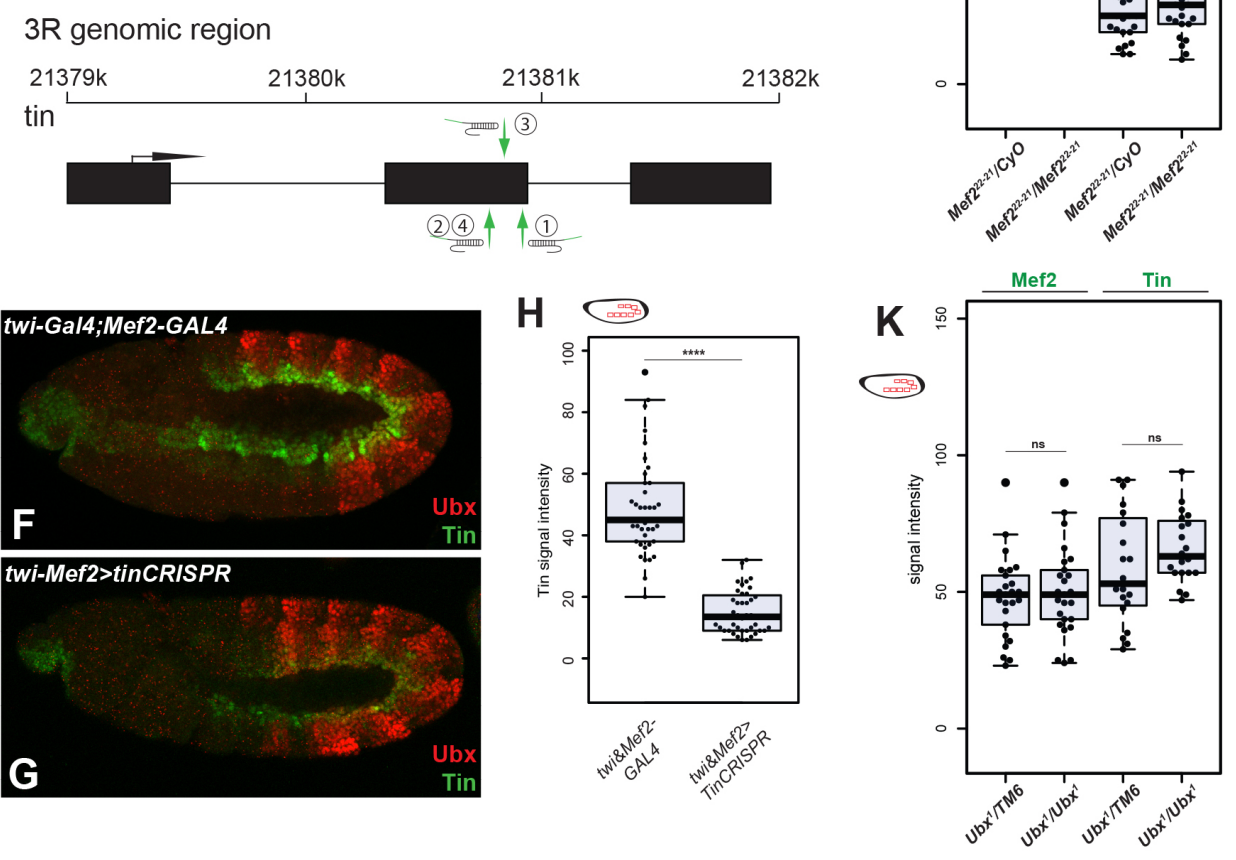

\section{SupFigure 3: Ubx genetically interacts with Tin but not with Mef2.}

A-D) Ubx, Tin and Mef2 mutants stage 11, stained with Ubx (red), Mef2 (green) and Tin (green). E) Localisation of the tin gRNA. F,G) Control (twi\&Mef2-Gal4) and tinCRISPR (twi\&Mef2>tinCRISPR) embryos stage 12 stained with Ubx (red) and Tin (green) antibodies, showing an decrease in Tin expression whereas Ubx expression remains. H) Validation of decreased Tin expression, illustration of the measured region in red (abdomen), ${ }^{* * * *} p=2.50 \mathrm{e}-$ 18. I,J,K) Validation of the protein expression from A-D indicated at the top of each panel, illustration of the measured region in red (abdomen) focusing on the somatic myoblasts, ns: non-significant. 

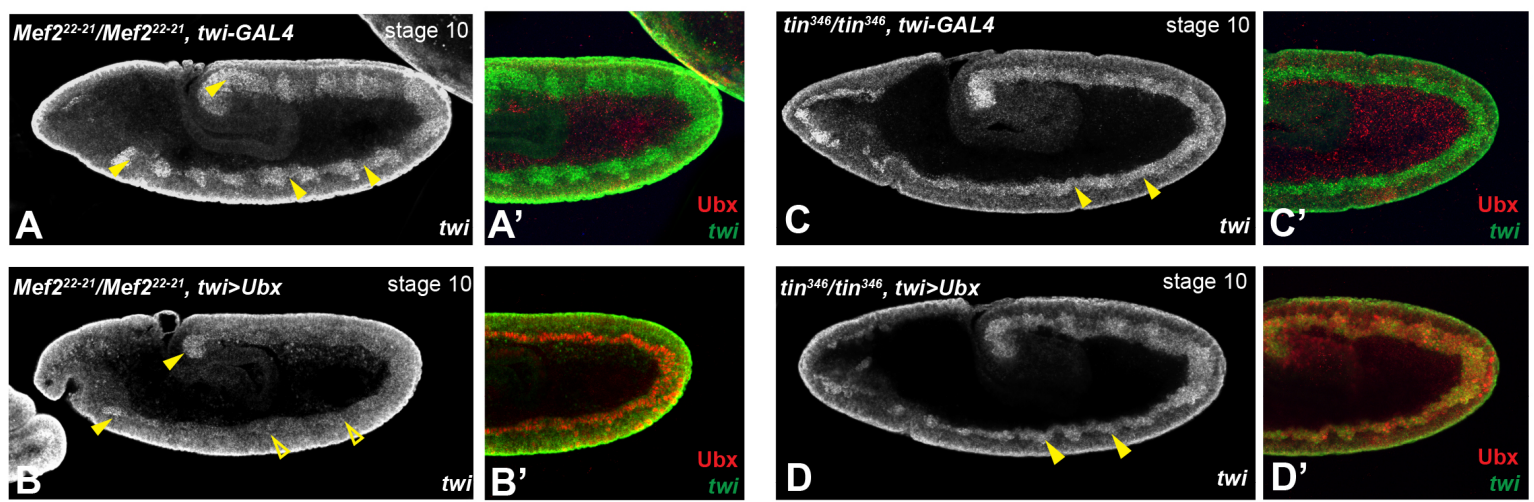

E

TinCRISPR

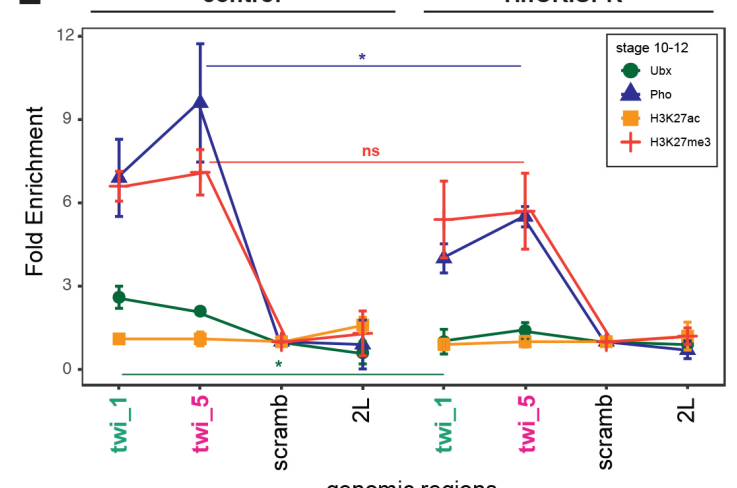

$\mathbf{F}$

control

UbxCRISPR

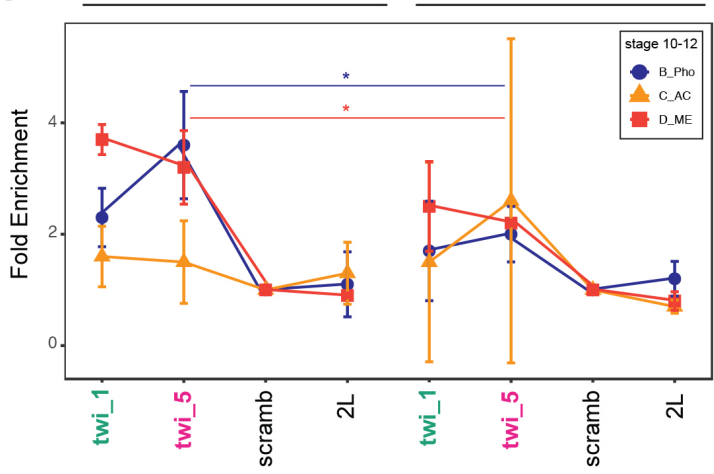

genomic regions

G

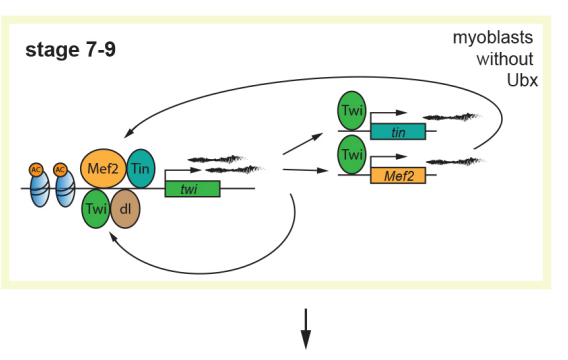

stage 14-17 myoblasts with Ubx

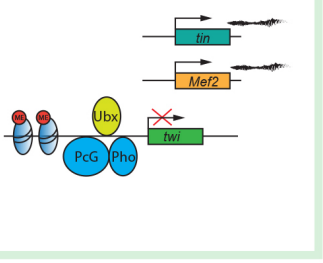

4

stage 10-12
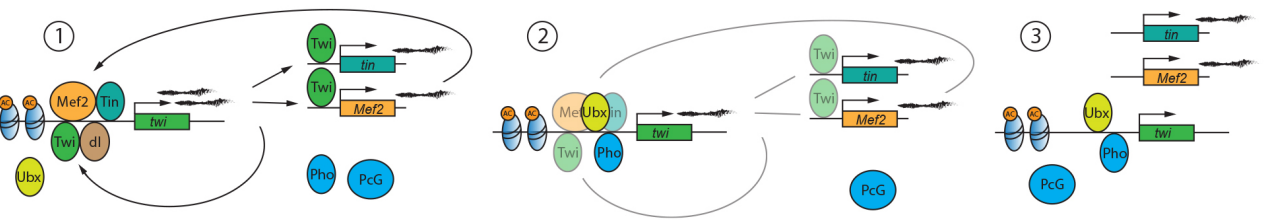

Figure 4: Ubx interaction with the twi promoter depends on Tin.

A-D) in situ hybridisation of control homozygous Mef2 and tin mutants containing the twi-GAL4 driver $(A, C)$ and Mef2 and tin homozygous mutants (B,D) overexpressing Ubx stage 10 using 
the twi probe (white, green) and Ubx antibody (red) stainings, showing that the twi expression is reduced (open yellow arrows) in Mef2 mutants overexpressing Ubx and is not affected in tin mutants overexpressing Ubx (closed yellow arrows). E) Ubx-ChIP (green), Pho-ChIP (blue), H3K27me3 (red) and H3K27ac (orange) experiments using control (twi\&Mef2-GAL4) and tinCRISPR (twi\&Mef2>tinCRISPR) chromatin at stage 10-12, indicating a decrease of Ubx (* $p=0.011)$ and Pho ( $\left.{ }^{*} p=0.029\right)$ binding to region 1 and 5 upon decreased Tin levels. H3K27me3 levels are decreased but not significant. H3K27ac levels are unaffected. F) Pho-ChIP (blue), H3K27me3 (red) and H3K27ac (orange) experiments using control (twi\&Mef2-GAL4) and UbxCRISPR (twi\&Mef2>UbxCRISPR) chromatin at stage 10-12, indicating a decrease of Pho ( ${ }^{*} p=0.0230$ ) binding and H3K27me3 levels $\left({ }^{*} p=0.050\right)$ to region 1 and 5 upon decreased Ubx levels. H3K27ac levels are slightly but not significantly increase. G) Predicted model, stage 79: Mef2 and Tin bind to the twi promoter and maintain its activity, stage 10-12: Ubx starts to be expressed in the mesoderm and replace Mef2, interacts with Tin and attracts Pho, the expression of twi gets reduced, stage 14-17: Ubx interact with Pho and attract PcG to maintain the inactivation. 


\section{References}

Akam, M., 1987. The molecular basis for metameric pattern in the Drosophila embryo, Development.

Atkins, M., Jiang, Y., Sansores-Garcia, L., Jusiak, B., Halder, G., Mardon, G., 2013. Dynamic Rewiring of the Drosophila Retinal Determination Network Switches Its Function from Selector to Differentiation. PLoS Genetics 9.

Azpiazu, N., Frasch, M., 1993. tinman and bagpipe: two homeo box genes that determine cell fates in the dorsal mesoderm of Drosophila.

Baker, R., Schubiger, G., 1996. Autonomous and nonautonomous Notch functions for embryonic muscle and epidermis development in Drosophila. Development 122, 617626.

Bate, M., Rushton, E., Currie, D.A., 1991. Cells with persistent twist expression are the embryonic precursors of adult muscles in Drosophila, Development.

Baylies, M.K., Bate, M., 1996. twist: A Myogenic Switch in Drosophila. SCIENCE 272.

Bischof, J., Maeda, R., Hediger, M., Karch, F., Basler, K., 2007. An optimized transgenesis system for Drosophila using germ-line-specific C31 integrases, PNAS.

Bour, B.A., O’Brien, M.A., Lockwood, W.L., Goldstein, E.S., Bodmer, R., Taghert, P.H., Abmayr, S.M., Nguyen, H.T., 1995. Drosophila MEF2, a transcription factor that is essential for myogenesis. Genes and Development 9, 730-741.

Brand, H., Perrimon, N., 1993. Targeted gene expression as a means of altering cell fates and generating dominant phenotypes. Development.

Busser, B.W., Gisselbrecht, S.S., Shokri, L., Tansey, T.R., Gamble, C.E., Bulyk, M.L., Michelson, A.M., 2013. Contribution of Distinct Homeodomain DNA Binding Specificities to Drosophila Embryonic Mesodermal Cell-Specific Gene Expression Programs. PLoS ONE 8.

Cripps, R.M., Black, B.L., Zhao, B., Lien, C.-L., Schulz, R.A., Olson, E.N., 1998. The myogenic regulatory gene Mef2 is a direct target for transcriptional activation by Twist during Drosophila myogenesis. Genes and Development.

Davis, T.L., Rebay, I., 2017. Master regulators in development: Views from the Drosophila retinal determination and mammalian pluripotency gene networks. Developmental Biology.

Domsch, K., Carnesecchi, J., Disela, V., Friedrich, J., Trost, N., Ermakova, O., Polychronidou, M., Lohmann, I., 2019. The Hox transcription factor Ubx stabilizes lineage commitment by suppressing cellular plasticityin Drosophila. eLife 8. 
Graham, A., Papalopulu, N., Krumlauf, R., 1989. The murine and Drosophila homeobox gene complexes have common features of organization and expression. Cell 367-78.

Huang, K., 2000. Solution structure of the MEF2A-DNA complex: structural basis for the modulation of DNA bending and specificity by MADS-box transcription factors. The EMBO Journal 19, 2615-2628.

Jiang, J., Kosman, D., Ip, Y.T., Levine, M., 1991. The dorsal morphogen gradient regulates the mesoderm determinant twist in early Drosophila embryos. Genes and Development.

Jin, H., Stojnic, R., Adryan, B., Ozdemir, A., Stathopoulos, A., Frasch, M., 2013. GenomeWide Screens for In Vivo Tinman Binding Sites Identify Cardiac Enhancers with Diverse Functional Architectures. PLoS Genetics 9.

Kremser, T., Hasenpusch-Theil, K., Wagner, E., Buttgereit, D., Renkawitz-Pohl, R., 1999. Expression of the b3 tubulin gene (b Tub60D) in the visceral mesoderm of Drosophila is dependent on a complex enhancer that binds Tinman and UBX. Mol Gen Genet 643658.

Leptin, M., 1991. twist and snail as positive and negative regulators during Drosophila mesoderm development.

Levine, M., Davidson, E.H., 2005. Gene regulatory networks for development.

Lewis, E.B., 1978. A gene complex controlling segmentation in Drosophila. Nature 276, 56570.

Liu, Y.H., Jakobsen, J.S., Valentin, G., Amarantos, I., Gilmour, D.T., Furlong, E.E.M., 2009. A Systematic Analysis of Tinman Function Reveals Eya and JAK-STAT Signaling as Essential Regulators of Muscle Development. Developmental Cell 16, 280-291.

Manak, J.R., Mathies, L.D., Scott, M.P., 1995. Regulation of a decapentaplegic midgut enhancer by homeotic proteins. Development 3605-3619.

Molkentin, J.D., Olson, E.N., 1996. Combinatorial control of muscle development by basic helix-loop-helix and MADS-box transcription factors.

Morata, G., Kerridge, S., 1982. The role of position in determining homoeotic gene function in Drosophila, Nature.

Pearson, J.C., Lemons, D., McGinnis, W., 2005. Modulating Hox gene functions during animal body patterning. Nature Reviews Genetics. https://doi.org/10.1038/nrg1726

Port, F., Bullock, S.L., 2016. Augmenting CRISPR applications in Drosophila with tRNAflanked sgRNAs. Nature Methods 13, 852-854. 
Port, F., Chen, H.M., Lee, T., Bullock, S.L., 2014. Optimized CRISPR/Cas tools for efficient germline and somatic genome engineering in Drosophila. Proceedings of the National Academy of Sciences of the United States of America 111.

R Core Team, 2018. R: A language and environment for statistical computing.

Ranganayakulu, G., Elliott, DA., Harvey, RP., Olson, EN., 1998. Divergent roles for NK-2 class homeobox genes in cardiogenesis in flies and mice. Development 125, 3037-3048.

Ray, R.P., Aroraf', K., Nusslein-Volhardt, C., Gelbart, W.M., 1991. The control of cell fate along the dorsal-ventral axis of the Drosophila embryo, Development.

Sandmann, T., Girardot, C., Brehme, M., Tongprasit, W., Stolc, V., Furlong, E.E.M., 2007. A core transcriptional network for early mesoderm development in Drosophila melanogaster. Genes and Development 21, 436-449.

Sanson, B., White, P., Vincent, JP., 1996. Uncoupling cadherin-based adhesion from wingless signalling in Drosophila, Nature.

Schindelin, J., Arganda-Carreras, I., Frise, E., Kaynig, V., Longair, M., Pietzsch, T., Preibisch, S., Rueden, C., Saalfeld, S., Schmid, B., Tinevez, J., White, D., Hartenstein, V., Eliceiri, K., Tomancak, P., Cardona, A., 2012. Fiji: an open-source platform for biological-image analysis. Nature Methods 9, 676-682.

Scott, M.P., Carroll, S.B., 1987. The Segmentation and Homeotic Gene Network in Early Drosophila Development Review, Cell.

Steiner, F.A., Talbert, P.B., Kasinathan, S., Deal, R.B., Henikoff, S., 2012. Cell-type-specific nuclei purification from whole animals for genome-wide expression and chromatin profiling. Genome Research 22, 766-777.

Struhl, G., 1982. Genes controlling segmental specification in the Drosophila thorax (homeosis/insect segments/determination/clonal analysis), Proc. NatL Acad. Sci. USA. Thisse, C., Perrin-Schmitt, F., Stoetxel, C., Thisse, B., 1991. Sequence-Specific Transactivation of the Drosophila twist Gene by the dorsal Gene Product.

Yin, Z., Xu, XL., Frasch, M., 1997. Regulation of the Twist target gene tinman by modular cis-regulatory elements during early mesoderm development. Development 124, 49714982. 